\title{
Integrated SIMS-AFM Instrument for Accurate High-Sensitivity and High- Resolution Chemical 3D Analysis
}

\author{
Y. Fleming, T. Wirtz, S. Eswara Moorthy
}

Advanced Instrumentation for Ion Nano-Analytics (AINA), Luxembourg Institute of Science and Technology, 41 rue du Brill, L-4422 Belvaux, Luxembourg

With the progress of miniaturization, driven by future needs in various fields in materials and life science, the 3D analysis of devices and material structures becomes increasingly challenging. As a consequence, the interest for performing bimodal or even multimodal nano-analysis has increased during the last decade. In particular, nano-analytical techniques and instruments providing both excellent spatial resolution and high-sensitivity chemical information are of utmost importance for investigations at the nanoscale.

Secondary Ion Mass Spectrometry (SIMS) is a method of choice for high sensitivity analysis. State-ofthe-art SIMS imaging instruments can provide chemical 2D and 3D maps with a lateral resolution of around 50nm. Other advantages of SIMS include excellent dynamic range and the ability to differentiate between isotopes and thus to map isotopic ratios. However, several important artifacts arise from the fact that the 3D mappings do not take into account the sample's surface topography. While the 3D reconstruction protocols and software assume that the initial sample surface is flat and the analyzed volume is cuboid, "real samples" present a surface topography, which furthermore changes during the ion bombardment as the local sputter yields depend on parameters such as the local angle of incidence of the ion beam and the crystal orientation. In addition, the situation is worsened if the sample is constituted of different materials due to preferential sputtering phenomena. As a consequence, the produced 3D images are affected by a more or less important uncertainty on the depth scale and can be distorted.

In order to obtain real high-resolution SIMS 3D analyses without being prone to the aforementioned artifacts, we developed a Scanning Probe Microscopy (SPM) module which we integrated into the Cameca NanoSIMS50 [1-4]. This in-situ combination between SIMS and SPM avoids the artifacts (e.g. different environment conditions, topography changes due to surface diffusion and reaction of reactive species used as primary ion sin SIMS) occurring when an ex-situ combination between these same techniques is used. In addition, this integrated instrument allows a combination of SIMS images with KPFM (Kelvin Probe Force Microscopy) data recorded in-situ in order to provide an extended picture of the sample under study. The known information channels of SIMS, AFM and KPFM are thus combined in one analytical and structural tool, enabling new multi-channel nanoanalytical experiments. This opens the pathway to new types of information about the investigated nanomaterials.

In this paper, we will illustrate the analytical potential of the combined SIMS-SPM approach by presenting several applications in the fields of materials science and life science.

\section{References:}

[1] Y. Fleming, T. Wirtz, U. Gysin, T. Glatzel, U. Wegmann, E. Meyer, U. Maier, J. Rychen, Appl. Surf. Sci. 258(4), 1322-1327 (2011). 
[2] T. Wirtz, Y. Fleming, U. Gysin, T. Glatzel, U Wegmann, E. Meyer, U. Maier, and J. Rychen, Surf Interface Anal. 45 (1), 513-516 (2013).

[3] T.Wirtz, Y. Fleming, M. Gerard, U. Gysin, T. Glatzel, E. Meyer, U. Wegmann, U. Maier, A. H. Odriozola and D. Uehli, Rev. Sci. Instrum. 83, 063702 (2012).

[4] C. L. Nguyen, T. Wirtz, Y. Fleming and J. B. Metson, Appl. Surf. Sci. 265, 489-494 (2013).

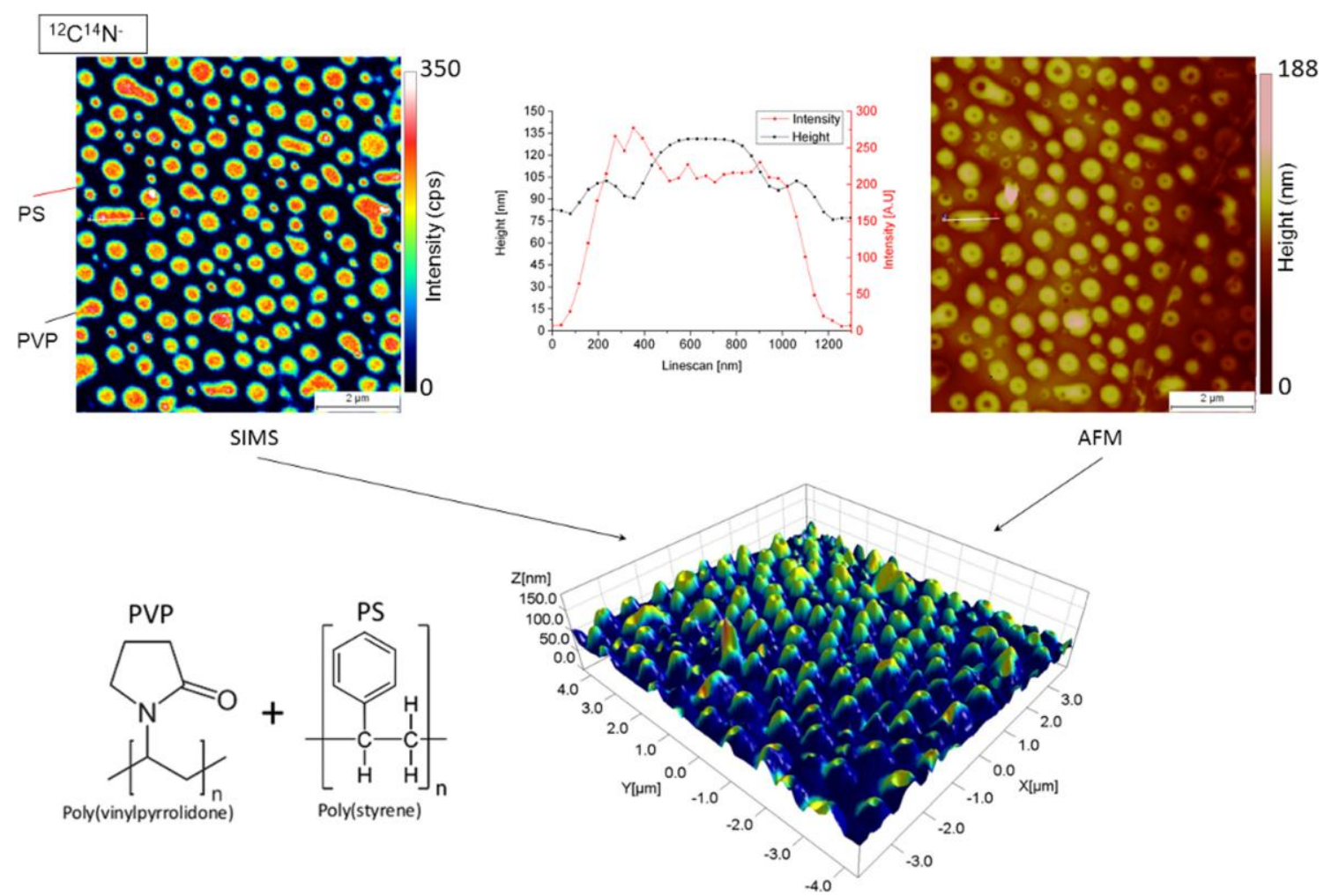

Figure 1. PVP/PS polymer blend after $\mathrm{Cs}^{+}$bombardment of $10^{16}$ ions $/ \mathrm{cm}^{2}$ : The SIMS recorded secondary ion intensity and the AFM recorded topography of the area of interest are superposed and compiled into a 3D surface mapping.

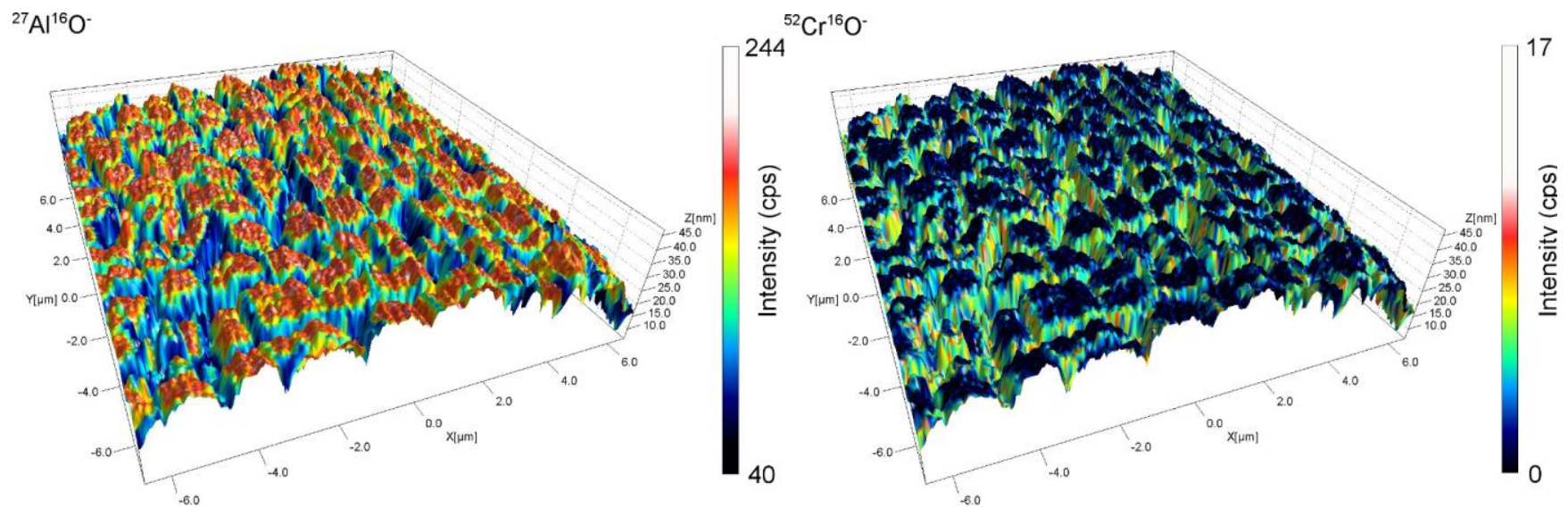

Figure 2. ${ }^{27} \mathrm{Al}^{16} \mathrm{O}^{-}$(left) and ${ }^{52} \mathrm{Cr}^{16} \mathrm{O}^{-}$(right) 3D SIMS-AFM images of a nickel-based super-alloy. The $3 \mathrm{D}$ images nicely show the correlations between the chemical composition and the topography. 